\title{
Efeito de soluções fluoretadas contendo xilitol e sorbitol no número de estreptococos do grupo mutans na saliva de seres humanos
}

\author{
Nilza Cristina Lopes Afonso de Valor Gonçalves, ${ }^{1,4}$ Aylton Valsecki \\ Júnior, ${ }^{2}$ Sérgio Luiz de Souza Salvador ${ }^{3}$ e Genevile Carife Bergamo ${ }^{4}$
}

RESUMO O objetivo do estudo foi avaliar o efeito de soluções de fluoreto de sódio a 0,05\% contendo 2,5\% ou $12,5 \%$ de xilitol no número de estreptococos do grupo mutans presentes na saliva. Participaram do estudo duplo cego, do tipo cruzado, 50 meninos entre 8 e 16 anos, distribuídos aleatoriamente em quatro grupos. Destes, 33 finalizaram o estudo. As soluções utilizadas foram: solução placebo; solução de fluoreto de sódio a 0,05\%; solução de fluoreto de sódio a $0,05 \%+2,5 \%$ xilitol $+2 \%$ sorbitol; solução de fluoreto de sódio a $0,05 \%+12,5 \%$ xilitol $+2 \%$ sorbitol. Os indivíduos utilizaram $20 \mathrm{~mL}$ de uma das soluções, duas vezes ao dia. Cada solução foi utilizada por um período experimental de 28 dias. Os períodos experimentais foram intercalados por períodos de descanso de 10 dias. As soluções contendo xilitol a 2,5\% e 12,5\% não apresentaram diferença significativa $(\mathrm{P}=0,32)$ em termos do logaritmo do número de estreptococos do grupo mutans. No entanto, a diferença foi significativa quando essas soluções foram comparadas às soluções de fluoreto de sódio e placebo $(\mathrm{P}<0,001)$. Os resultados sugerem que a solução de fluoreto de sódio a 0,05\% com adição de xilitol a 2,5\% ou 12,5\% reduziu significativamente o número de estreptococos do grupo mutans.

Palavras-chave Cárie dental, agentes cariostáticos, fluoretos, xilitol, sorbitol.

Os produtos utilizados para higiene bucal geralmente contêm substâncias com ação antimicrobiana. Os agentes

\footnotetext{
1 Universidade Estadual de São Paulo (UNESP), Faculdade de Odontologia de Araraquara, Mestrado em Odontopediatria, Araraquara, SP, Brasil. Correspondência e pedidos de separatas devem ser enviados a Nilza C.L.A.V. Gonçalves no seguinte endereço: Rua Coronel Francisco A. Coutinho no. 4, apto. 7, CEP 13025-190, Campinas, SP, Brasil. E-mail: nilzavalor@yahoo.com

2 UNESP, Faculdade de Odontologia de Araraquara, Departamento de Odontologia Social, Araraquara, SP, Brasil.

3 Universidade de São Paulo (USP), Faculdade de Ciências Farmacêuticas de Ribeirão Preto, Departamento de Análises Clínicas, Toxicológicas e Biomatológicas, Ribeirão Preto, SP, Brasil.

4 Universidade de Alfenas, Alfenas, MG, Brasil.
}

antimicrobianos aplicados profissionalmente ou sob a forma de dentifrícios e soluções de bochecho podem reduzir a incidência de cárie mediante controle da formação de placa, suprimindo espécies cariogênicas, ou mediante inibição do metabolismo bacteriano (1).

O flúor tem seu principal efeito anticárie sobre o esmalte, mas pode, também, ter um importante, porém sutil, efeito antimicrobiano (1). Assim, o flúor pode não causar uma alteração direta na composição da microbiota, mas pode atuar preservando a homeostase microbiana na placa e estabilizando-a durante as condições de oscilação de concentração de açúcar e mudança de $\mathrm{pH}(2)$.

Um dos mecanismos pelos quais a homeostase do ambiente bucal pode ser interrompida é através da exposição da placa ao baixo $\mathrm{pH}$, devido à freqüente ingestão de carboidratos fermentáveis (3). A prevenção da doença pode estar relacionada à redução da freqüência dos desafios ácidos. Isso pode ser obtido tanto pela simples diminuição de alimentos contendo carboidratos fermentáveis quanto pelo consumo de alimentos contendo açúcares alternativos, os quais não podem 
ser ou são fracamente metabolizados pelas bactérias da placa.

Os açúcares substitutos mais utilizados em produtos divulgados como benéficos à saúde bucal, em comparação aos açúcares contendo sacarose, são sorbitol, manitol e xilitol. Os açúcares alcoólicos têm demonstrado propriedades não cariogênicas ou cariogenicidade extremamente baixa, visto estudos com ratos e alguns estudos clínicos realizados em seres humanos (4).

Os estudos relacionados à utilização do xilitol tiveram início na década de 70. Nesses estudos, a substituição total de sacarose por xilitol na dieta demonstrou redução significativa da incidência de cárie $(5,6)$. Com base em tais resultados, gomas de mascar e outros produtos contendo xilitol foram avaliados em populações de vários países. Os dados clínicos reunidos sugeriram que o consumo de xilitol poderia estar associado a uma grande redução do incremento de superfícies cariadas, perdidas e obturadas (CPOS) em indivíduos jovens e adultos (7).

$\mathrm{O}$ xilitol tem sido incorporado principalmente em gomas de mascar e dentifrícios e, recentemente, em soluções para bochecho. Os resultados dos estudos com dentifrícios contendo xilitol, em relação a seus efeitos sobre a saliva, placa dental e incidência de cárie, são contraditórios (8-12). Por outro lado, estudos in vitro e in vivo têm sugerido que a associação de fluoreto e xilitol apresenta propriedades anti-cárie adicionais quando comparada à utilização do fluoreto apenas $(10,11,13-16)$. Os efeitos de soluções para bochecho contendo xilitol sobre a placa dental e a saliva são bem menos estudados (17-21).

Frente ao exposto, o objetivo deste estudo foi verificar o efeito de soluções fluoretadas contendo xilitol e sorbitol sobre o número de unidades formadoras de colônias (UFC) de estreptococos do grupo mutans na saliva de indivíduos com idade entre 8 e 16 anos.

\section{MATERIAIS E MÉTODOS}

Foram incluídos no estudo 50 indivíduos do sexo masculino, com idade entre 8 e 16 anos, internos em uma ins-

FIGURA 1. Fluxograma do delineamento experimental, estudo sobre o efeito de soluções fluoretadas contendo xilitol e sorbitol no número de estreptococos do grupo mutans, Araraquara $(\mathrm{SP})$, Brasila

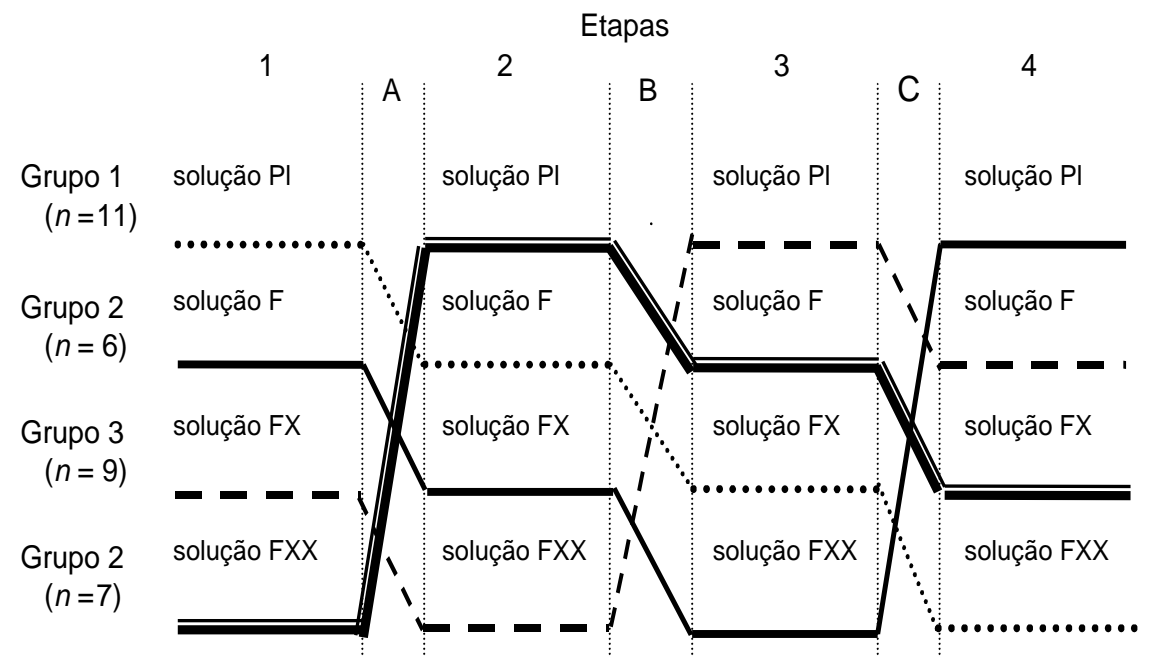

a Etapa 1: dia 1 a 28; etapa 2: dia 39 a 66; etapa 3: dia 77 a 104; etapa 4: dia 115 a 142 (final do experimento). A, B, C = períodos de descanso de 10 dias entre as etapas. $\mathrm{PI}=$ solução placebo de flúor/xilitol/sorbitol; $\mathrm{F}$ = solução de fluoreto de sódio a $0,05 \% ; F X=$ solução de fluoreto de sódio a $0,05 \%+2,5 \%$ xilitol $+2 \%$ sorbitol; $F X X=$ solução de fluoreto de sódio a $0,05 \%+12,5 \%$ xilitol $+2 \%$ sorbitol

tituição pública para menores órfãos (população total da instituição). Esclarecimentos relacionados aos riscos e benefícios frente à utilização do regime de soluções para bochecho foram apresentados. $O$ protocolo foi aprovado pelo responsável pela Instituição, assim como pela Secretaria da Saúde do Município de Araraquara, São Paulo, Brasil.

O delineamento foi do tipo duplo cego, cruzado 4X4. Os indivíduos foram divididos aleatoriamente em quatro grupos (figura 1). As soluções para bochecho utilizadas no estudo foram: solução placebo de flúor/xilitol/sorbitol (Pl); solução de fluoreto de sódio a $0,05 \%(\mathrm{~F})$; solução de fluoreto de sódio a $0,05 \%+2,5 \%$ xilitol $+2 \%$ sorbitol (FX); solução de fluoreto de sódio a $0,05 \%+12,5 \%$ xilitol $+2 \%$ sorbitol (FXX). Os indivíduos utilizaram $20 \mathrm{~mL}$ de uma das soluções duas vezes ao dia, após o café da manhã e à noite, antes de dormir, com o objetivo de expor o ambiente bucal a uma concentração diária de $1 \mathrm{~g}$ ou $5 \mathrm{~g}$ de xilitol. Os bochechos foram supervisionados pela primeira autora deste trabalho. Cada solução foi utilizada durante um período experi- mental de 28 dias. Os períodos experimentais foram intercalados por períodos de descanso de 10 dias. Ao final do estudo - ou seja, ao final de 142 dias todos os participantes tinham sido submetidos aos quatro tratamentos.

\section{Avaliação do número de unidades formadoras de colônias de estreptococos do grupo mutans}

Amostras de saliva não estimulada foram coletadas nos períodos anteriores e posteriores a cada etapa do estudo, totalizando oito amostras por indivíduo ao final do estudo. As coletas foram realizadas pela primeira autora deste estudo, sempre no período da manhã, antes do horário do café da manhã $(23,24)$. Foram utilizados tubos estéreis de boca larga $(20 \times 150 \mathrm{~mm})$. Os tubos foram acondicionados em uma caixa de isopor e resfriados em banho de gelo até o momento do processamento da saliva, sem que esse período excedesse 4 horas.

As amostras de saliva foram, primeiramente, homogeneizadas com o auxílio de um aparelho de ultra-som, 
em potência máxima, durante 10 segundos. O meio de cultura SB20, idealizado por Davey e Rogers (25), foi utilizado por ser seletivo para estreptococos do grupo mutans. O volume de $0,05 \mathrm{~mL}$ de saliva pura ou das diluições de cada amostra foi semeado em placas de vidro. As placas foram transferidas para jarras de anaerobiose, em atmosfera com $10 \%$ de $\mathrm{CO}_{2}$ e incubadas a $37{ }^{\circ} \mathrm{C}$ durante 72 horas. Após esse período, o número de UFC de estreptococos do grupo mutans foi determinado com o auxílio de um microscópio estereoscópico.

Para a análise estatística, os dados foram submetidos a uma transformação logarítmica a fim de assegurar uma normalidade (Shapiro-Wilk). A análise de variância para um fator (one-way) foi aplicada para verificar a possibilidade de agrupar os indivíduos distribuídos nos diferentes grupos do estudo cruzado. Além disso, a análise de variância e o teste de Newman-Keuls foram utilizados para comparar o efeito das soluções a partir do número médio de UFC (log) de estreptococos do grupo mutans proveniente da diferença final e inicial em cada solução de bochecho.

\section{RESULTADOS}

Dos 50 indivíduos inicialmente incluídos no estudo (população total da instituição), 33 concluíram o experimento (66\%); portanto, 17 indivíduos (34\%) desistiram de participar. Os motivos que levaram à desistência incluem necessidade de tratamento odontológico ou utilização de antibióticos durante o período do estudo, desligamento da instituição ou falta de colaboração para realização do regime de bochechos.

Constatou-se, pelo teste de ShapiroWilk, que os dados referentes ao número de estreptococos do grupo $m u$ tans, após transformação logarítmica, não diferiam de uma distribuição normal.

Os indivíduos distribuídos entre os quatro grupos do estudo cruzado foram agrupados de acordo com o tipo de solução, independentemente da etapa em que a mesma foi utilizada, uma vez que, pelas análises da variância, não se observou diferença entre as etapas considerando cada uma das soluções (Pl: $P=0,55 ; \mathrm{F}: P=0,42 ; \mathrm{FX}: P=$ 0,12; e FXX: $P=0,68)$.

A comparação entre as quatro soluções de bochecho foi realizada com base na diferença entre o logaritmo do número de UFC de estreptococos do grupo mutans no período posterior $\mathrm{e}$ anterior às administrações de cada uma das soluções de bochecho (análise da variância: $P<0,0001)$. Os resultados da aplicação do teste NewmanKeuls aparecem na tabela 1.

A tabela 1 mostra que a utilização das soluções F, FX e FXX diferiu significativamente da utilização da solução placebo $(P<0,0001)$. As soluções FX e FXX apresentaram diferenças significativas $(P=0,001$ e $P<0,0001$, respectivamente) quando comparadas à solução $\mathrm{F}$, porém não apresentaram diferença significativa entre si $(P=0,32)$.

As soluções de fluoreto de sódio a $0,05 \%$ com $2,5 \%$ ou $12,5 \%$ de xilitol apresentaram melhores resultados em relação à diminuição do número de estreptococos do grupo mutans por $\mathrm{mL}$ de saliva quando comparadas à solução de fluoreto de sódio a 0,05\%. Esses resultados sugerem, portanto, que a associação de flúor e xilitol promove um efeito adicional na redução do número de estreptococos do grupo mutans, embora esse efeito não apresente alterações com o aumento da concentração de xilitol.

TABELA 1. Médias das diferenças do logaritmo do número de unidades formadoras de colônias de estreptococos do grupo mutans no período anterior e posterior à utilização de cada uma das soluções para bochecho, Araraquara (SP), Brasil

\begin{tabular}{lr}
\hline \multicolumn{1}{c}{ Soluções } & Médias $^{\mathrm{a}}$ \\
\hline Placebo & 0,44935 \\
Flúor $0,05 \%$ & $-0,36288$ \\
Flúor $0,05 \%+$ xilitol $2,5 \%+$ & \\
$\quad$ sorbitol $2 \%$ & $-0,89759^{*}$ \\
Flúor $0,05 \%+$ xilitol $12,5 \%+$ & $-1,06078^{*}$ \\
$\quad$ sorbitol $2 \%$ & \\
\hline
\end{tabular}

a Por $\mathrm{ml}$ de saliva. As médias seguidas de asterisco não diferem entre si $(P=0,32)$ pelo teste de Newman-Keuls.

\section{DISCUSSÃO}

Os estudos relacionados à utilização do xilitol na dieta, em substituição à sacarose, tiveram início na década de $70(5,6)$. Desde então, o xilitol vem sendo adicionado em gomas, dentifrícios e soluções para bochecho.

O xilitol não é fermentado pela maioria dos microorganismos bucais e, portanto, é considerado não cariogênico, por não promover a formação de produtos finais ácidos pelas bactérias da placa dental (26-28). Propriedades anti-cárie ou terapêuticas também têm sido atribuídas ao xilitol $(6,27,29)$. Outros efeitos do xilitol incluem diminuição dos níveis salivares de estreptococos do grupo mutans (26-28, 30-32) e diminuição da formação de placa dental (26-28, 31-33).

A concentração de xilitol capaz de promover alterações na cavidade bucal varia em cada estudo. Estudos clínicos e microbiológicos de substituição parcial da sacarose utilizando concentrações de xilitol próximas a 10 g por dia demonstraram diminuição no nível de estreptococos do grupo mutans na saliva e na placa, além de redução na quantidade de placa e índice de CPOS $(15,31)$. Loesche et al. (30), utilizando gomas de mascar, observaram que pequenas quantidades de xilitol (5g por dia) resultaram em diminuição significativa dos níveis de Streptococcus mutans na saliva e na placa dental. Kandelman e Gagnon (22) observaram que $0,8 \mathrm{~g}$ por dia de xilitol foram suficientes para diminuir significativamente a progressão da cárie após o período de 12 meses, em comparação à não utilização de xilitol adicionado em goma. Além disso, o mesmo estudo sugeriu que o aumento da concentração de xilitol $(3,4 \mathrm{~g}$ por dia) intensificaria seu efeito em relação à progressão da cárie dental (22).

No presente estudo, a utilização de $20 \mathrm{ml}$ de solução de fluoreto de sódio a $0,05 \%$ com adição de $2,5 \%$ ou $12,5 \%$ de xilitol, duas vezes ao dia, proporcionou a exposição do ambiente bucal a uma concentração diária de $1 \mathrm{~g}$ ou $5 \mathrm{~g}$ de xilitol, respectivamente. Nossos resultados mostraram que ambas as concentrações foram associadas a uma 
diminuição significativa dos níveis salivares de estreptococos do grupo $\mathrm{mu}$ tans; esta observação vai ao encontro dos resultados obtidos em outro estudo (30), no qual $5 \mathrm{~g}$ por dia de xilitol promoveram diminuição significativa dos níveis salivares de estreptococos do grupo mutans.

Os resultados do presente estudo sugerem que a associação de flúor e xilitol promove um efeito adicional na redução do número de estreptococos do grupo mutans quando comparada com a utilização do flúor apenas; contudo, o aumento da concentração de xilitol não promoveu uma intensificação de efeito, ao contrário do que foi sugerido por estudos anteriores $(14,22)$.

A grande maioria dos estudos realizados até o presente momento enfocou a utilização do xilitol através de gomas de mascar. Um aspecto que deve ser ressaltado é que existe, sem dúvida, uma tendência para preconizar o uso de goma de mascar com xilitol como meio preventivo à cárie dental. Entretanto, o uso da goma por si só não constitui uma medida preventiva que alcança resultados significativos com relação ao controle da placa e da cárie, pelo menos em comparação aos meios tradicionais; a goma deve ser utilizada em ocasiões especiais, quando a escovação ou outros meios mecânicos ou químicos de controle da placa estejam impossibilitados (34). Dessa forma, a utilização de dentifrícios e soluções para bochecho contendo xilitol deve ser levada em consideração. É importante ressaltar que, após o período de 10 dias sem utilização da solução para bochecho, os níveis salivares de estreptococos do grupo mutans retornam a seus valores iniciais, o que sugere que o xilitol deve ser utilizado de forma contínua.

Frente a essas observações, a utilização de soluções de fluoreto de sódio a $0,05 \%$ contendo $2,5 \%$ ou $12,5 \%$ de xilitol pode ser recomendada como coadjuvante dos procedimentos mecânicos de higiene bucal, principalmente em indivíduos com alto risco para a doença da cárie, embora estudos de longa duração devam ser realizados com o intuito de avaliar o efeito dessas soluções a longo prazo. Além disso, estudos adicionais são necessários a fim de elucidar o mecanismo de ação do xilitol associado ao flúor, assim como para avaliar o efeito da redução do número de microorganismos e da acidogenicidade bacteriana na desmineralização do esmalte dental.

\section{REFERÊNCIAS}

1. Marsh PD. Antimicrobial strategies in the prevention of dental caries. Caries Res 1993;27(1): 72-76.

2. Marsh PD. Sugar, fluoride, $\mathrm{pH}$ and microbial homeostasis in dental plaque. Proc Finn Dent Soc 1991;87(4):515-525.

3. Bradshaw DJ, McKee AS, Marsh PD. Effects of carbohydrate pulses and $\mathrm{pH}$ on population shifts within oral microbial communities in vitro. J Dent Res 1989;68(9):1298-1302.

4. Imfeld T. Efficacy of sweeteners and sugar substitutes in caries prevention. Caries Res 1993;27(1):50-55.

5. Mäkinen KK, Scheinin A. Turku sugar studies. VI. The administration of the trial and the control of the dietary regimen. Acta Odontol Scand 1975;33(70):105-127.

6. Scheinin A, Mäkinen KK, Ylitalo K. Turku sugar studies V. Final report on the effect of sucrose, fructose and xylitol diets on the caries incidence in man. Acta Odontol Scand 1975;33(70):67-104.

7. Makinen KK, Makinen PL, Pape HR Jr, Peldyak J, Hujoel P, Isotupa KP, et al. Conclusion and review of the "Michigan Xylitol Programme" (1986-1995) for the prevention of dental caries. Int Dent J 1996;46(1):22-34.

8. Cutress T, Howell PT, Finidori C, Abdullah F. Caries preventive effect of high fluoride and xylitol containing dentifrices. J Dent Child 1992;9(4):313-318.

9. Makinen KK, Söderling E, Hurttia H, Lehtonen OP, Luukkala E. Biochemical, microbiologic, and clinical comparisons between two dentifrices that contain different mixtures of sugar alcohols. J Am Dent Assoc 1985;111(5): 745-751.
10. Petersson LG, Birkhed D, Gleerup A, Johansson M, Jönsson G. Caries-preventive effect of dentifrices containing various types and concentrations of fluorides and sugar alcohols. Caries Res 1991;25(1):74-79.

11. Sintes JL, Escalante C, Stewart B, McCool JJ, Garcia L, Volpe AR, Triol C. Enhanced anticaries efficacy of a $0.243 \%$ sodium fluoride/ $10 \%$ xylitol/silica dentifrice: 3-year clinical results. Am J Dent 1995;8(5):231-235.

12. Svanberg M, Birkhed D. Effect of dentifrices containing either xylitol and glycerol or sorbitol on mutans streptococci in saliva. Caries Res 1991;25(6):449-53.

13. Arends J, Smits M, Ruben JL, Christoffersen J. Combined effect of xylitol and fluoride on enamel demineralization in vitro. Caries Res 1990;24(4):256-257.

14. Gaffar A, Blake-Haskis JC, Sullivan R, Simone A, Schmidt R, Saunders F. Cariostatic effect of a xylitol/ $\mathrm{NaF}$ dentifrice in vivo. Int Dent J 1998;48(1):32-39.

15. Isokangas $P$, Alanen $P$, Tiekso J, Mäkinen KK. Xylitol chewing gum in caries prevention: a field study in children. J Am Dent Assoc 1988;117(2):315-320.

16. Rogers AH, Bert AG. Effect of xylitol and fluoride on the response to glucose pulses of Streptococcus mutans T8 growing in continuous culture. Oral Microbiol Immunol 1992; 7(2):124-126.

17. Giertsen E, Emberland H, Scheie AA. Effects of mouth rinses with xylitol and fluoride on dental plaque and saliva. Caries Res 1999; 33(1):23-31.

18. Lingstrom $P$, Lundgren F, Birkhed D, Takazoe I, Frostell G. Effects of frequent mouthrinses with palatinose and xylitol on dental plaque. Eur J Oral Sci 1997;105(2):162-169.

19. Mühlemann HR, Schmidt R, Noguchi T, Imfeld T, Hirsch RS. Some dental effects of xylitol under laboratory and in vivo conditions. Caries Res 1977;11(5):263-276.

20. Paunio K, Hurttia H, Tenovuo J, Mäkinen KK, Tiekso J. Effects on oral health of mouthrinses containing xylitol, sodium cyclamate and sucrose sweeteners in the absence of oral hygiene. I. Clinical findings and analysis of gingival exudate. Proc Finn Dent Soc 1984;80(1): 3-12.

21. Tenovuo J, Mäkinen KK, Paunio K. Effects on oral health of mouthrinses containing xylitol, sodium cyclamate and sucrose sweeteners in the absence of oral hygiene. IV. Analysis of whole saliva. Proc Finn Dent Soc 1984;80(1): 28-34.

22. Kandelman D, Gagnon G. Clinical results after 12 months from a study of the incidence and progression of dental caries in relation to consumption of chewing-gum containing $\mathrm{xy}-$ litol in school preventive programs. J Dent Res 1987;66(8):1407-1411.

23. Krasse B. Caries Risk: A practical guide for assessment and control. Chicago: Quintessence Publ Co, 1985.

24. Matsukubo T, Saito H, Ohta K, Maki Y, Sazuka I, Takaesu Y, et al. A practical method for differentiating the salivary levels of Streptococcus mutans using a stabilized selective broth. Bull Tokyo Dent Coll 1983;24(4): 195-202.

25. Davey AL, Rogers AH. Multiple types of the bacterium Streptococcus mutans in the human mouth and their intra-family transmission. Archs Oral Biol 1984;29(6):453-460. 
26. Birkhed D. Cariologic aspects of xylitol and its use in chewing gum: a review. Acta Odontol Scand 1994;5(2):116-127.

27. Tanzer JM. Xylitol chewing gum and dental caries. Int Dent J 1995;45(1):65-76.

28. Trahan L. Xylitol: a review of its action on mutans streptococci and dental plaque - its clinical significance. Int Dent J 1995;45(1):77-92.

29. Havenaar R. The anti-cariogenic potential of xylitol in comparison with sodium fluoride in rat caries experiments. J Dent Res 1984;63(2): 120-123.

30. Loesche WJ, Grossman N, Earnest R, Corpron $R$. The effect of chewing xylitol gum on the plaque and saliva level of Streptococcus mutans. J Am Dent Assoc 1984;108(4):587-592.

31. Söderling E, Mäkinen KK, Chen CY, Pape Jr HR, Loesche W, Mäkinen PL. Effect of sorbitol, xylitol, and xylitol/sorbitol chewing gum on dental plaque. Caries Res 1989;23(5): 378-384.

32. Soderling E, Trahan L, Tammiala Salonen T, Häkkinen L. Effects of xylitol, xylitol-sorbitol, and placebo chewing gums on the plaque of habitual xylitol consumers. Eur J Oral Sci (Denmark) 1997;105(2):170-177.

33. Larmas M, Scheinin A, Gehring F, Mäkinen KK. Turku sugar studies XX - microbiologi- cal findings and plaque index values in relation to 1-year use of xylitol chewing gum. Acta Odontol Scand 1975;33(70):321-336.

34. Oliveira AGRC, Costa ICC, Silva PR, Moimaz SAA. Ação da goma de mascar com xilitol sobre o $\mathrm{pH}$ da placa bacteriana após ingestão de sobremesa à base de sacarose. RPG 1998;5(1):7-12.

Manuscrito recebido em 16 de junho de 2000. Aceito em versão revisada em 30 de novembro de 2000

ABSTRACT The objective of this study was to assess the effect of $0.05 \%$ sodium fluoride solutions containing $2.5 \%$ or $12.5 \%$ xylitol on the number of Streptococcus mutans in the human mouth. Fifty boys between 8 and 16 years of age participated in this double-blind

Effect of mouth rinses containing xylitol and sorbitol on the number of Streptococcus mutans in the human mouth crossover study. Of the original 50 boys, 33 finished the study. Participants were randomly divided into four groups. The following solutions were employed: placebo solution; $0.05 \%$ sodium fluoride solution; $0.05 \%$ sodium fluoride $+2.5 \%$ xylitol $+2 \%$ sorbitol; $0.05 \%$ sodium fluoride $+12.5 \%$ xylitol $+2 \%$ sorbitol. Each solution was used for a 28 -day period $(20 \mathrm{~mL} /$ day, twice a day), with a 10 -day washout period between solutions. There were no significant differences $(P=0.32)$ between the two xylitolcontaining solutions ( $2.5 \%$ vs. $12.5 \%$ ) concerning the number of Streptococcus mutans. However, there was a significant difference between these two xylitol-containing solutions and the sodium fluoride and placebo solutions $(P<0.001)$. Our results suggest that the $0.05 \%$ sodium fluoride solutions containing either $2.5 \%$ or $12.5 \%$ xylitol caused a significant reduction in the number of Streptococcus mutans.

For a very long time we have accepted the old adage "Prevention is better than cure." In our new era, the belief in it-for of its truth there can be no doubt-must be made ever more manifest in our research and its directives and in our teaching. The most conspicuous interest of the student ten or twenty years hence will, I hope, no longer be in the rare or difficult and too often incurable case, but in the common and more understandable and preventable disease. May the daily question on this lips become not "What is the treatment?" but "What are the causes?"and "If preventable, then why not prevented?"

[Por mucho tiempo hemos aceptado el antiguo refrán "prevenir es mejor que curar". En nuestra nueva era, la creencia en él (porque no hay duda de su veracidad) debe hacerse más patente aun en nuestras investigaciones y sus directrices, así como en nuestra enseñanza. Dentro de 10 ó 20 años, el interés principal del estudiante, así lo espero, ya no radicará en el caso insólito o difícil y muchas veces incurable, sino en las enfermedades comunes más manejables y evitables. Ojalá que su pregunta diaria ya no sea “¿cuál es el tratamiento?" sino “¿cuáles son las causas?” "y ¿si es prevenible, entonces por qué no tomar medidas preventivas?".]

John A. Ryle, Changing Disciplines, 1948 